\title{
Tactical Communications between Military Vehicles
}

\author{
André Filipe da Silva Ferreira, Maria João Martins, João Paulo N. Torres, and António Baptista
}

\section{ABSTRACT}

The increasing evolution and development of telecommunications as well as the need for wider bandwidths to comply with the users' needs, has led to the development of the so-called free space optical communications. The advantages of optical communications in comparison to radio frequency communications draw interest for certain military applications.

This paper describes the simulation, development and implementation of an optical communication system which integrates the various functional blocks of the optical emitter and the optical receiver and presents some theoretical considerations about the specific characteristics of the propagation of the optical signals in free space. The implementation of this system in military vehicles to allow wider bandwidth in military communications, as well as to function as an alternative system to the used systems, is one of the objectives of this dissertation.

For this purpose, two circuits (emitter and receiver) were projected and designed including the design of printed circuit boards (PCB) and performed some simulations of the optical part of the complete system and the electronic simulation of some parts of the electronic circuits.
Published Online: January 10, 2021

ISSN: $2684-4451$

DOI :10.24018/ejphysics.2021.3.1.42

André Ferreira

Academia Militar, Lisboa, Portugal. João Torres*

Instituto Superior Técnico and Academia Militar, Lisboa, Portugal.

(e-mail: joaoptorres@ hotmail.com) Maria Martins

Academia Militar, Lisboa, Portugal. António Baptista

Instituto Superior Técnico, Lisboa, Portugal.

*Corresponding Author
Keywords: Military, Communication, Vehicles.

\section{INTRODUCTION}

The evolution of technology has always accompanied armed conflicts in the world. Many inventions emerged as a direct consequence of the two world wars. Radar, tanks, and even the discovery of the nuclear bomb and its impact were major inventions that gave its users an advantage over enemies. We can affirm in these situations that they allowed the countries that had them to have clear advantage over all their opponents.

The concept of information-seeking dates to the period of ancient China when Sun Tzu, a brilliant General and Chinese strategist, argued that information was crucial to gaining an advantage over an opposing force in a given strategic situation [1].

In this way, we can infer that telecommunication plays a fundamental role in the defense of each country. In addition, the technological means are constantly evolving, and the latest inventions allow information to be obtained in an increasingly rapid and credible way. Thus, by using technologies, it is possible to detect, eliminate or cause casualties in the enemy, increasingly, with the minimum commitment of our forces.

The concept of time becomes very important in a conflict. Thus, the faster the information is disseminated, the faster the decisions can be taken by the command, which will make the actions/missions that the militarized forces perform in the different theaters of operation more likely to succeed [2].

This means that the military needs media that allow the upward flow of information to the commander and descendant flow towards the subordinates to facilitate the necessary coordination on the battlefield of the different missions during the battle. In addition, it is necessary that there be different programs that integrate and concentrate information in a central body (high military ranks) to enable the two essential functions in the Army, Command and Control (C2).

Thus, the objective of this dissertation is the development of an optical communication system for implementation in the military vehicles, and to accomplish this objective it is necessary: to carry out a previous study of the optical communication systems in the world; explain the framework of the theme in the current panorama in the Portuguese Army; to carry out the determination of the system parameters taking into account all the limitations of the project and to validate all the work done through system block simulations as well as system-wide simulations.

\section{THE STATE OF ART}

\section{A. Optical Communication System}

An optical communication system presents in its constitution numerous functional blocks, with the fundamental objective of exchanging information between two terminals, usually referred to as transmitter and receiver.

The amount of information that can be transmitted in any communication system is directly related to the bandwidth of the signal sent, which in turn directly relates to its working frequency. Thus, when optical signals are used in the communication, it is possible to guarantee very high 
transmission rates since these signals have frequencies in the order of THz [3].

Free Space Optics (FSO) means the transmission of information using optical signals through the atmosphere between two points.

For example, the laser in the emitter is responsible for the conversion of the signal sent from the electrical domain to the optical domain. At the receiver, the signal is picked up by a photodetector, which converts the optical signal to electric, passing to the next block, which is usually the preamplification stage of the signal until it reaches the output of the receiver where all the information is obtained from the transmitter, assuming that there were no errors in the whole process (neither in transmitter, nor in transmission nor in receiver) [4].

\section{B. International Projects with FSO Systems}

Exists several international projects that applies FSO technology. The objective of this subchapther is to present projects of optical communication systems that have been developed all over the world, explaining the principles of operation of each system, as well as the differences between each one of them.

\section{a) Car $2 x$-vehicle Information and communication system}

Japanese researchers have developed several projects in this area, of which the following stand out: the Car $2 x$ project, where several optical components are used for the communication of information from car to car, or else from car to infrastructure.

In the case of this system, a component called Light Emitting Diode (LED) is used which is responsible for sending the emitter signal, while on the other side of the communication system a silicon Positive Intrinsic Negative (PIN) photodiode is used, which is responsible for receiving the signal emitted. The place for installation of the system can be, as already mentioned within vehicles or in infrastructures, and this choice varies depending on the functionalities for which the components have been configured.

The Japanese project called Vehicle Information and Communication System (VICS) is an innovative information and communication system in which users receive traffic information in real time. In brief, the information is processed in a data processing center, and is made available to users through the navigation screen of their vehicles [5].

This information can have three levels in this system:

- $\quad$ Level 1 - Plain text;

- Level 2 - Simple diagrams;

- Level 3 - Graphical information through a navigation system.

This system allows collecting and transmitting information on traffic, traffic lights, unoccupied parking places, or even on road accidents quickly for users equipped with the transceiver.

The distribution of this information to users is done by two ways: using radio lights, normally installed on motorways, which emit in the microwave range, having a cover with this form of transmission about 200 kilometers from the vehicle transceiver; or by using the so-called infrared headlamps which are installed along the various roads in Japan. They receive the information sent to them by the traffic control center, which in turn relays that information to all vehicles with an information area of 30 kilometers [6].

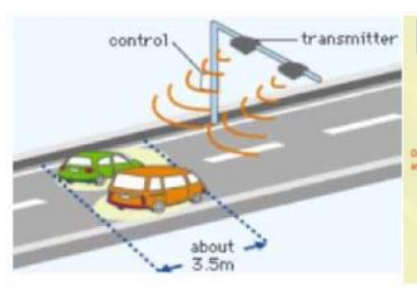

(a)

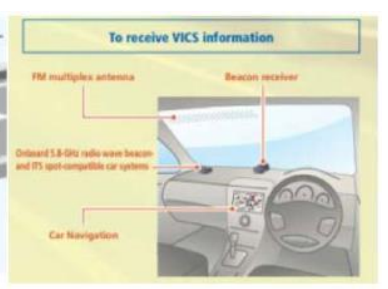

(b)
Fig. 1. (a) Infrared headlight information distribution [6]; (b) Typical car mount equipped with VICS [6].

\section{b) Co-operative networks for intelligent road safety}

Another project that addresses the same theme in a similar way but is being developed by several European countries, is the project called Cooperative Networks for Intelligent Road Safety (COOPERS).

The operation of this system is very similar to that used in the VICS system. An optical transceiver is added to each vehicle, which makes it possible to receive messages with information from anyone road unit of this system. In the same way, the car also sends information to road units, what makes this system bidirectional.

All the information is sent to a central information processing service also called COOPERS Service Center.

After processing all the information, events (accidents, malfunctions, etc.) are created in the Traffic Control Center, which is the infrastructure responsible for disseminating the events to all users, using the system's road units [7].

\section{c) Visible light communication - Li-Fi}

Another project capable of providing optical communication between users is Visible Light Communication (VLC). For this, it makes use of lowcost equipment, and for the transmission of information can use two technologies: either using LEDs, or photodiodes, as in the case of Car2x [8].

There are numerous applications that make use of this technology. Light Fidelity is a wireless communication system with high bi-directional transmission speed. It can also be used for communication between vehicles and for communication between vehicles and infrastructures. This technology has been associated with the theme Internet of Things, in which everything is connected to the internet using LEDs as access points. The exchange of information is done by turning the beam of each LED on and off, with a periodicity in the order of nanoseconds to become imperceptible to the human eye [9].

\section{Attenuation in the Propagation in FSO Systems}

The attenuation of the atmosphere is defined as the process by which part of the energy of the electromagnetic wave is lost when crossing the atmosphere. Thus, the atmosphere causes degradation and attenuation of the signal in an FSO system connection in various ways: absorption, dispersion, and turbulence. There are also the geometric losses of the path which is the quotient between the surface 
area of the receiving lens, by the area of the surface of the beam that reaches the lens in the receiver. Since the beam is divergent the surface area of the beam increases as we move away from the emitter [10], [11].

All these effects vary over time and depend on local conditions and climate. In general, the attenuation of the atmosphere is given by the following equation (Beer's law):

$$
l_{a}=l_{\text {geom }} * \exp (-\alpha d)
$$

where $l_{a}$ is the attenuation introduced into the atmosphere, $l_{\text {geom }}$ are the geometric losses, $d$ is the distance in kilometers between the terminals. $\alpha$ is the total attenuation coefficient and is given by:

$$
\alpha=\alpha_{\text {abs }} \alpha_{\text {scat }}
$$

where $\alpha_{a b s}$ is the attenuation coefficient due to the molecular absorption in the aerosols and $\alpha_{\text {scat }}$ is the attenuation coefficient due to the molecular dispersion in the aerosols.

$$
l_{\text {geom }}=\frac{d_{2}^{2}}{\left|d_{1}+(d \theta)\right|^{2}}
$$

In this case, $d_{2}$ is the aperture diameter of the receiving lens in meters, $d_{1}$ diameter of the aperture of the emission lens in meters, $\theta$ is the angle of divergence of the optical beam in milliradians and $d$ is the distance of the connection in meters [10].

Typically, geometric losses in an optical communication system are limiting, hence it is necessary to use a component complementary to the photoemitter, usually a signal collimator, to reduce losses, which increases the efficiency of the communication process.

Thus, the performance of our system can be described by the power flow, where $p_{R}$ is the power of the received signal and $p_{T}$ is the emitted power, through the expression:

$$
p_{R}=p_{T} \cdot l_{\text {geom }} \exp (-\beta d)=p_{T} l_{a}
$$

The minimum required power that the signal must have, for the optical receiver to detect it in good condition, is called the receiver sensitivity $(S r)$ [dBm]. Sensitivity depends essentially on system noise sources, such as ambient light and electronic noise, which can influence signal detection. Knowing the power received on a logarithmic scale $\left(P_{R x}, d B m\right)[\mathrm{dBm}]$, we obtain the safety margin of a connection, which is very important to ensure the operation of our system, regardless of variables external to the system, namely disturbances due to propagation in free space [11].

$$
M_{\text {ligação }}[d B]=P_{R x, d B m}-S_{r} .
$$

Generally, a connection margin of $10 \mathrm{~dB}$ is used as an optimum operating value of the systems.

\section{CONTEXT IN THE PORTUGuese ARMY}

The digitization of the modern battlefield has empowered the C4I systems (command, control, communications, computers, and information) that require the treatment and the rapid sharing of information at the various levels to aid in decision making. Consequently, the provision of a greater amount of information and better quality of services (voice, data, and image), requires more robust, efficient, and higher bandwidth communications to provide the necessary information to users' requirements [12].

In this context, the Portuguese Army has been constantly making a great effort to modernize its C4I capacity, through a substantial investment in communications and information systems (CSI). From the upper tactical ranks (brigade or superior), through the intermediate and lower tactical ranks, which act at the front of the battle, allowing rapid bidirectional dissemination of information, to foster decisionmaking at various levels and a more rapid performance of forces [12].

\section{A. Information and Communications System - Tactical (ICS-T)}

The ICS-T is the Army's response, in its tactical component, to emerging obligations and the need to conduct network-centric operations. As part of the process of transformation and modernization of the Army, it was designed to respond to the evolution of the operational environment, but also to the growing expansion of networks and sources of information, whose complexity implies compliance with NATO Network Enabled Capability. These standards aim to allow the existence of robust, networkcentric organizations to increase the capacity for information sharing, which increases the quality of information and allows a more precise and effective knowledge of the operational situation at each moment of time, through the Common Operational Picture (COP) [13].

This network-centric environment improves collaboration across all levels of the Army's organizational chart and further enhances the ability to synchronize between the various actors which allows for speeding up the designated command speed, which is the time taken to make decisions depending on each situation. The final stage of this cycle is reflected in a continuous increase in capacity and in greater efficiency in carrying out the mission [12].

The ICS-T is divided into two subsystems:

- Communications Subsystem, which integrates the TICS, Combat Net Radio (CNR) and C4I components of the Soldier Combat Systems (SCS);

- Information Subsystem, which integrates C2 support systems, namely the Army Command and Control Information System (SICCE), the Battlefield Management System (BMS) and the Dismounted Soldier System Command and Control (DSS-C2).

\section{a) Communications subsystem}

The Communications Subsystem implements a CSI infrastructure that integrates all existing entities in the battlefield, and this infrastructure must ensure interconnection between decision makers, sensors, and weapon systems, materializing the communications network of a "Centralized Networked Force" [12]. 
It is defined by law that Portugal undertakes to support, if necessary, with a brigade unit, or else three battalions, the North Atlantic Treaty Organization (NATO). As such, the means of support by the ICS-T were considered considering this situation, being possible to divide the communication subsystem into two components: a conjunctural component and a structural component [14].

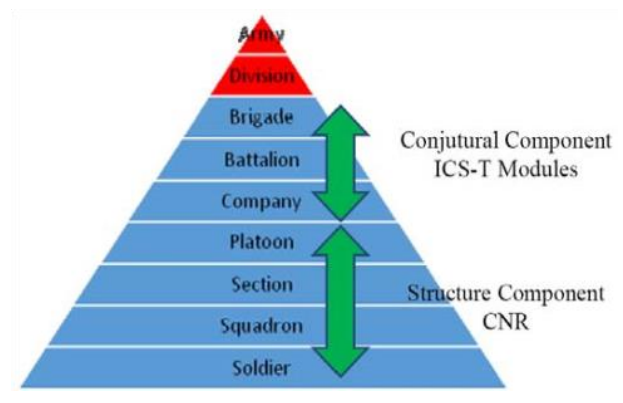

Fig. 2. Organization of the Army for the projection of Task Force and articulation with the Communications Subsystem.

In addition, the tactical communications conjunctural component consists of the communications subsystem that is based on a network structure with a modular structure based on ICS-T modules, with the following functions:

Implement the core tactical communications network;

- Establish the connection between CP;

- Integrate mobile users of the radio networks into the main network.

\section{b) Conjunctural component}

To achieve the objectives imposed on conjunctural component, it was necessary to articulate the ICS-T modules and subdivide them into five subdivisions:

Extended Area Subsystem (EAS). Is the core of the network and integrates the Transit Node (TN) and RearLink (RL) modules, consisting of a set of switching nodes interconnected through multichannel radio links (hertzian bundles). Occasionally, broadband satellite terminals may be used.

Local Area Subsystem (LAS). Provides various services (voice, data, messages, facsimile, or video) to users, usually located at a Command Post (CP) of a certain tactical rank and guarantees access to the upper structure of the network through access nodes. LAS guarantees the necessary support flexibility to conduct an operation, regardless of the tactical rank (Brigade, Battalion or Company), consisting of the Access Node (AN), the Battalion Communication Center (BCC) and the Company Communication Center (CCC), the Brigade Command Staff (BrigCS) and Battalion Command Staff (BatCS) modules.

Mobile Users Subsystem (MUS). Is intended to support dispersed mobile users in the operations area and can be integrated into the tactical network through Radio Access Points (RAP) and, exceptionally, through the CCB and CCC modules. MUS integrates the PAR module and CNR users.

Network Security Subsystem (NSS). Is transversal to the entire structure of the ICS-T and implements different levels of security in the networks [for example: RED (secure network) and BLACK (non-secure network)].

Network Management Subsystem (NMS). Is based on a modular architecture based on the Network Management
Center that constitutes the "brain" of the communications subsystem.

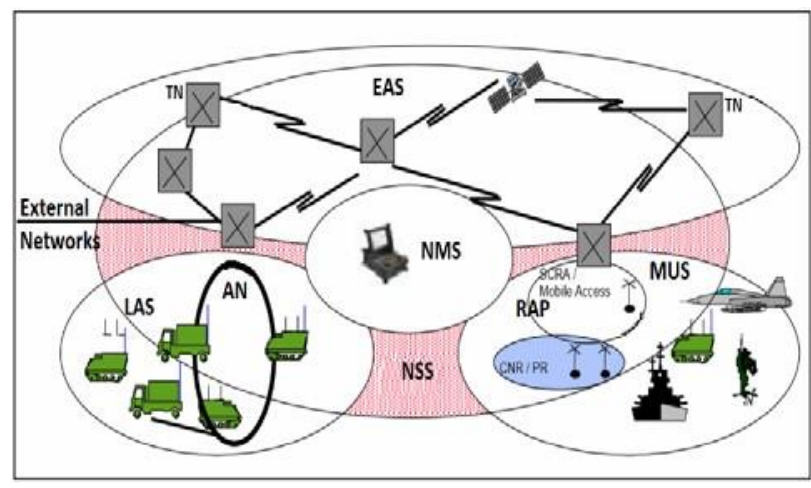

Fig. 3. Interconnections between ICS-T subsystems [12].

\section{c) SIC-T modules}

As explained above, for the implementation of the SIC-T functional architecture, eight systemic tactical modules were defined, which are responsible for the implementation of the tactical networks in the Portuguese Army.

The tactical modules are: Transit Node, Access Node, Radio Access Point, Rear-Link, Battalion Communication Center, Company Communication Center, Brigade Command Staff, and the Battalion Command Staff.

\section{Transit node}

The constitution of the TN is materialized by a shelter transported by a light tactical car. This module integrates the SAE and materializes a node, of the network structure that forms the backbone of the ICS-T.

The TN is based on a hertzian beam infrastructure that guarantees the automatic routing of the information flow through a set of redundant connections (establishment up to four connections), able to ensure the survival of the system.

\section{Access node}

The constitution of the AN is materialized by two shelters carried by two medium/heavy tactical vehicles. This module integrates the LAS and is intended to provide users of the $\mathrm{CP}$ of a Brigade, with a set of communications media and application services, necessary to support the command and control action of the respective commander.

The complexity underlying the $\mathrm{AN}$ and the tactical considerations require its distribution in two shelters, one of Transmission and one of C2 \& Management. The radiant means are placed in the Transmission shelter in a sufficiently high position to allow the best propagation conditions. In addition, it must be installed at a good distance from the $\mathrm{C} 2$ shelter, to minimize the possibility of radiolocation of the $\mathrm{CP}$. The Transmission shelter has the capacity to establish up to three radio link connections, which together with the availability of optical fiber, become the main means of integrating this module into the upper network structure (SAE). In the C2 \& Management shelter lies the ability to make teleservices available to CP users, usually in a tent or in containers located in the immediate vicinity. Inside this shelter, the military are responsible for the network management, applications, and services available to users.

\section{Radio access point}

The RAP must be installed in a tactical vehicle that has the same tactical mobility as the brigade supported. The 
main function of the RAP is the integration of the GRC-525 radio in the environment of a "full IP" network, through calls:

- Voice dialing for the IP network automatically;

- Selective to the radio;

- And a data service with tactical intranet implementation.

This IP/Radio integration in the RAP is implemented in order to make the system more flexible, since it allows to dynamically assign the radios to the voice or data service (tactical intranet).

This module is intended to create the conditions for establishing the interface structure between MUS and EAS.

This interface is ensured by the installation of a set of communications equipment with a strong radio component (VHF/UHF/HF) and a means component to integrate the upper network structure, mainly through radio and optical interfaces. A set of applications ensures the transfer of information flows between these systems.

The Pandur CV vehicle materializes a RAP establishing the links with the tactical modules.

\section{Rear-link}

The RL is installed in a light tactical shelter vehicle and has a tacit trailer that transports the base infrastructure to the HF component.

It can support an operational force, when projected outside the national territory, or ensure the integration of this same force with the fixed/ operational component (materialized by SIC-Op) when this is not possible with other means. It guarantees the connection to the rear (national territory), however it can be used, although with some limitations, to connect two transit nodes via satellite in conventional operations.

The RL has a satellite communications capability with broadband transmitter/receiver terminals, has the capability to use commercial civilian satellites or satellites made available by NATO operating on the $\mathrm{Ku}$ and $\mathrm{X}$ bands of the electromagnetic spectrum.

This module further provides an HF component, especially provided with two strands: the first, to ensure multiplicity of means for consolidating the rear connection, and the second, to ensure the connections, when necessary, over large distances within the area of supported force operations. The RL can be used in conjunction with another SIC-T module or alone because of the residual capacity of switching and processing of information that it has.

\section{Battalion communication center}

The BCC is designed to equip command offices with Battalion/Group units with the means of communication and the information systems necessary to support the command action of their commanders.

This module materializes in two shelters (C2 \& Management and Transmission), carried by two light/medium tactical vehicles.

\section{Company communication center}

The CCC is designed to provide the CP of the Company/Squad/Battery step units with communications and information systems required to support the command action of their commanders.

The company module will be built for a light tactical platform, regardless of the tactical unit for which it is intended, presenting a design adapted to the need for airborne projection.

In the construction of the CCC a very light and high mobility structure was adopted, compatible with the characteristics of the subunit for which it is intended. This is a single shelter to be installed in Light Wheel Tactical Car. This type of shelter, besides allowing the installation of communications equipment, has also integrated an energy component (generator) and masts.

\section{Center, brigade command staff and the battalion command staff}

Finally, the modules Brig CS and Bat CS are materialized in a set of commercial terminal equipment (telephones, printers, computers, projector, etc.), transported in robust boxes specially created for storage and transport (by land, air, and sea), giving the additional protection to these equipment's.

This equipment allows commanders and staff elements to plan and execute their operational missions.

\section{B. Employment Scenarios of the FSO System}

One of the characteristics necessary for evolution in a network is its scalability, and in the case of a tactical network, it must possess this capacity to allow to accommodate more users within the same tactical module. Which means that some of the connections must be modified to allow this. Thus, with this dissertation is intended to dimension and build an optical communication system, which will increase the capacity of the network between different modules.

Thus, I will introduce a new connection option in the ICS-T support concept, which brings innumerable advantages in its use, so we can establish a set of FSO application scenarios.

\section{a) Communication within the RAP (static situation)}

The purpose of the RAP is to establish a network coverage area in which operations can be developed. Thus, it is static for a certain period while there is a need to conduct operations in the area.

Pandur CV vehicles are used in the conduct of operations, with the purpose of guaranteeing communication within the Company, and as in the case of RAP, are also static during the execution of some operations.

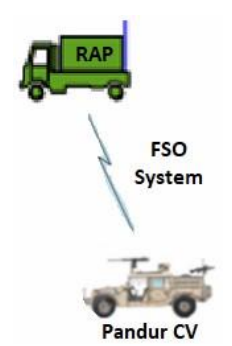

Fig. 4. Connection in RAP with Pandur CV.

Thus, we can use the FSO system to establish the communication channel between the different vehicles providing greater bandwidth in the communications and in the services made available by RAP.

\section{b) Re-transmitter on a mountain (static situation)}

FSO system can also be used as a signal relay, i.e., in the presence of a natural obstacle to communication we can 
placed a vehicle equipped with the system to ensure communication between different ranks.

For example, when there is a mountain obstructing communication between $\mathrm{CCB}$ and $\mathrm{CCC}$, it may be necessary to place a vehicle at the top of the mountain to ensure communication between the different forces.

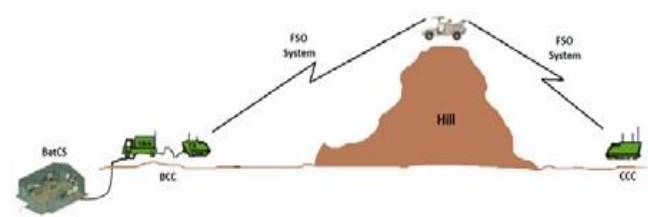

Fig. 5. Re-transmitter on a mountain.

\section{c) Communication between two combat vehicles}

The FSO system can be used in the process of two moving vehicles. That is, using a PAT system it is possible to establish communication during the movement of the vehicles. In this way, we can transmit information during the conduct of operations, which is advantageous in the sense that we are constantly in contact between the actors in the operations.

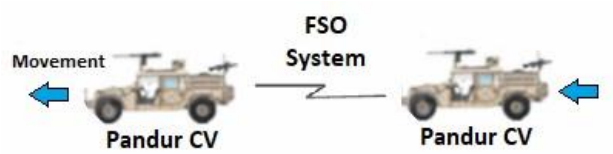

Fig. 6. Connection between two Pandur CV during a dislocation.

\section{BUILDING AN OPTICAL COMMUNICATION SYSTEM}

\section{A. Optical Transmitter}

The electrical scheme chosen to make the data transmission is adapted from an existing circuit, implemented with the use of two integrated circuits, and whose objective is to constitute itself as an optical data emitter in free space [15], [16].

The MAX3643 is an integrated circuit with the dimension of $4 \mathrm{~mm}$ by $4 \mathrm{~mm}$, and with the constitution of 24 pins that allow to control the excitation current of the laser. As for the integrated circuit DS1865 is a controller that checks the light output from the laser and adjusts the excitation current to keep the light output constant.

As we can see in Fig. 7 shows us the circuit that allows the transmission of information with these integrated circuits, for the high frequencies.

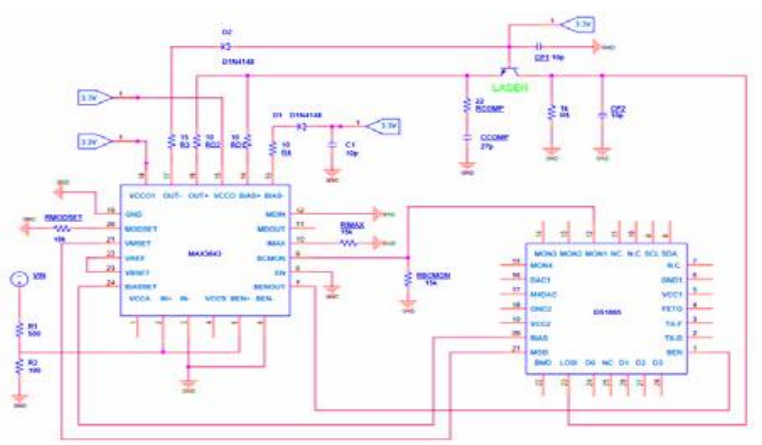

Fig. 7. Circuit with integrated components of the optical emitter.

Regarding the sizing of the optical emitter, the recommendations in the specifications of the integrated MAX3654 were followed, as well as the adaptation made in another work, adapting it to the requirements of my optical emitter [15], [16].

There are several components (resistors, capacitors, and diodes) that surround the MAX3643 integrated circuit, to allow the sizing of the currents, voltages, and operating frequencies of the optical emitter circuit.

The resistors $R_{1}$ and $R_{2}$ establish a voltage divider applied to the input signal $\left(V_{I N}\right)$, which allows the protection of both the integrated circuit and the laser. The signal coming from this voltage divider is the input signal on the integrated MAX3643, more specifically on the IN+ and BEN+ pins. While the $\mathrm{IN}+$ pin is responsible for accepting information bits, the $\mathrm{BEN}+$ pin (when active) is responsible for allowing the laser to emit light. In this case, the IN+ and BEN+ pins are connected to the same point on the circuit, meaning that when the $V_{I N}$ input signal is low, the laser is off. When the $V_{I N}$ input is at the high level, the BEN+ input allows the excitation of the laser according to the sizing done on the MODSET and BCMON pins, as will be seen later [15], [16].

The maximum input voltage on the $\mathrm{IN}+$ pin according to the specifications of the integrated is $0.8 \mathrm{~V}$, using Expression 6 to obtain the relation between resistors $R_{1}$ and $R_{2}$ :

$$
\left.V_{I N}=\left(R_{2} / R_{2}+R_{1}\right)\right) V_{I N}
$$

For this, the same rectangular pulse was considered for the input signal $V_{I N}$, but for calculation effect only its maximum value, $5 \mathrm{~V}$. Since the relation between $V_{I N}+$ and $V_{I N}$ is 0.16 , the dimensioning of the resistors was made considering $100 \Omega$ for $R_{2}$ and $500 \Omega$ for $R_{1}$ (an approximation of the exact value of $R_{1}-560 \Omega$ ) [15], [16].

Considering the specifications of the chosen laser, he has a threshold current value $\left(I_{t h}\right)$, which varies between $0.8 \mathrm{~mA}$ and $3 \mathrm{~mA}$ and operating current between $7 \mathrm{~mA}$ and $12 \mathrm{~mA}$. The maximum current at the output of the integrated MAX3643 on the $I_{\text {MAX }}$ pin $\left({ }^{I}\right.$ MAX $)$ is given by:

$$
I_{M A X}=I_{B I A S}+I_{M O D}
$$

The modulation current $I_{M O D}$. and the bias current $I_{B I A S}$ are switched off if its sum exceeds the limit defined by the IMAX pin resistance $\left(R_{I M A X}\right)$. As show in Fig. 8 , the characteristic curve of the OPV310Y laser and the currents addressed [15], [16].

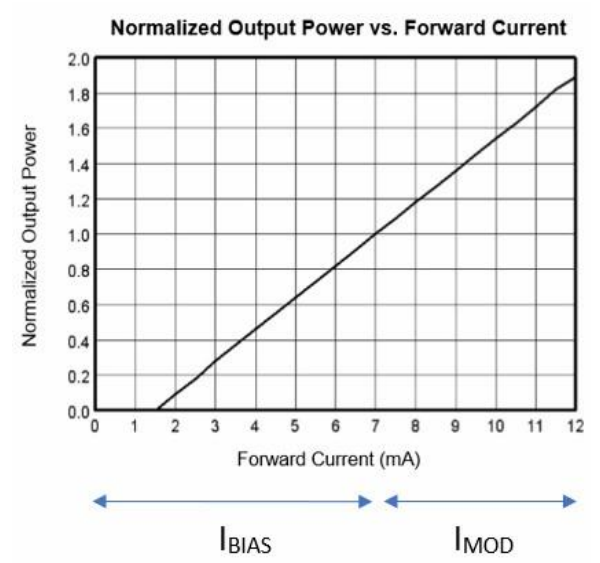

Fig. 8. Characteristic curve of the OPV310Y laser [17] 
By the observation of the figure, it is understood that the polarization current - $I_{\text {BIAS }}$ is a constant value that places the laser operating in the linear region and in a range beyond its threshold value (threshold current $-I_{t h}$ ).

On the other hand, the modulation current is switched on and off in synchronization with the input voltage waveform. Therefore, for the current $I_{B I A S}$ the value of $7 \mathrm{~mA}$ has been chosen and for the current $I_{M O D}$ the value of $5 \mathrm{~mA}$. It is now possible to control the maximum current at the controller output by properly choosing the value of $R_{\text {IMAX }}$ [15], [16].

According to the MAX3643 controller specifications, the value of $R_{I M A X}$ is done using Table 1 and an estimate of the value, considering the value of the minimum and maximum current of the controller.

TABLE 1: CURRENT LIMIT SPECIFICATIONS AT CONTROLLER OUTPUT

\begin{tabular}{|c|c|c|}
\hline $\begin{array}{c}\text { Resistance } R_{\max } \\
(\mathbf{k} \boldsymbol{\Omega})\end{array}$ & $\begin{array}{c}\text { Corrente } I_{\text {MAx }} \\
\text { minimum }(\mathbf{m A})\end{array}$ & $\begin{array}{c}\text { Corrente } \\
\text { maximum }\end{array}$ \\
\hline $\mathbf{3}$ & 155 & - \\
\hline $\mathbf{5}$ & 100 & 150 \\
\hline 10 & 50 & 75 \\
\hline
\end{tabular}

According to Table 1 , for a current $I_{\mathrm{MAX}}$ of $12 \mathrm{~mA}$ it is estimated the use of a resistance in the $I_{\text {MAX }}$ pin of $39 \mathrm{k} \Omega$, reducing the possibility of burning the laser. However, by imposition of the manufacturer, the maximum value for $R_{\text {IMAX }}$ is $15 \mathrm{k} \Omega$ being this the value that I used in the dimensioning of resistance $R_{\text {IMAX }}$ [15].

To control both the modulation current $I_{M O D}$ and the bias current $I_{B I A S}$, two resistors, respectively $R_{M O D S E T}$ and $R_{B C M O N}$ are introduced into the circuit.

The resistance value $R_{M O D S E T}$ can be obtained, according to the specifications of the controller MAX3643, through Expression 7.

$$
R_{M O D S E T}=1.2 \times\left(G_{M O D} / I_{M O D}\right)-R_{M O D}
$$

where $R_{M O D}$ and $G_{M O D}$, according to the manufacturer's specifications, are typically $50 \Omega$ and $88 \mathrm{~mA} / \mathrm{mA}$ and respectively mean the internal resistance of the MODSET pin and the modulation current gain [15], [16].

The resistance value $R_{M O D S E T}$ must be chosen to produce the maximum modulation current for the operating temperature of the chosen laser. Considering a modulation current ${ }^{I} M O D$ of $5 \mathrm{~mA}$, the resistance value $R_{M O D S E T}$ is $21.07 \mathrm{k} \Omega$ Thus, the resistance $R_{M O D S E T}$ is assumed to be $18 \mathrm{k} \Omega$, which will allow a modulation current $I_{M O D}$ of $5.85 \mathrm{~mA}$.

The resistance $R_{B C M O N}$ is obtained by knowing the bias current gain, $G B S M$, and that the voltage at its terminals, $V_{B C M O N}$, must be less than $1.4 \mathrm{~V}$. The resistance $R_{B C M O N}$ is determined, according to the specifications of the controller MAX3643, through Expression 8.

$$
R_{B C M O N}=V_{B C M O N} /\left(I_{B I A S} \mathrm{X} G_{B S M}\right)
$$

For a current $I_{B I A S}$ of $7 \mathrm{~mA}$ and with a gain $G_{B S M}$ of $14 \mathrm{~mA} / \mathrm{mA}$ a resistance $R_{B C M O N}$ of $14.29 \mathrm{k} \Omega$ is obtained. Therefore, for the resistance $R_{B C M O N}$ is considered the value of $15 \mathrm{k} \Omega$ which will allow a current $I_{B I A S}$ of $6.66 \mathrm{~mA}$, higher than the threshold current ( $3 \mathrm{~mA})$.
The OUT+ pin and the BIAS+ pin are the pins responsible for the output of the modulation and polarization currents respectively. Connected to these pins, in series, a damping resistance, $R_{D}\left(R_{D 1}\right.$ for the pin OUT + and $R_{D 2}$ for the pin BIAS+), increases the resistance to the passage of current in the laser. The sum of the value of this resistance with the equivalent laser resistance, $R_{\text {laser }}$, should be approximately $15 \Omega$. Since the typical resistance of a VCSEL laser varies from $4 \Omega$ to $6 \Omega$, its impedance is considered $5 \Omega$, which results in a resistance $R_{D 1}$ and $R_{D 2}$ of $10 \Omega[23]$.

The pins OUT- and BIAS- allow the output of the modulation current and the bias current respectively when the input of the BEN pin is at the low level. These current deviations cause the OUT-pin to be connected to a resistor of $15 \Omega\left(R_{3}\right)$ and a switching diode (diode $\left.1 \mathrm{~N} 4148\right)$ to the laser node and the BIAS- pin connected to a resistance of $10 \Omega\left(R_{4}\right)$, a diode $1 \mathrm{~N} 4148$ and a capacitor $(\mathrm{C} 1)$ of $10 \mathrm{pF}$ to $V_{\mathrm{CC}}(3.3 \mathrm{~V})$, not allowing the passage of current in this direction [15], [16].

At high frequencies, as in the case, we must also introduce an RC connection $\left(R_{C O M P}\right.$ and $\left.C_{C O M P}\right)$ connected, in parallel, between the laser cathode and the earth, to reduce possible distortions in the duty cycle of the laser, caused by parasitic inductances. In this circuit the value of $22 \Omega$ was used for $R_{\text {СОMP }}$ and for $C_{\text {СОMP }}$ of $27 \mathrm{pF}$, which results, considering Expression 9, at a cut-off frequency of 267.9MHz [15], [16].

$$
X_{C}=1 / 2 \pi f_{c} C
$$

Finally, the printed circuit design was implemented in the Eagle program, version 9.0.1, with the objective of producing a Printed Circuit Board (PCB). The Eagle enables the drawing of the electric circuit in scheme (.sch) and after it is finished, generate the project in printed board format (.brd).

A printed circuit board supports and establishes electrical connections between electrical components using conductive tracks. As for the components, they are soldered onto the board to secure them, bringing advantage in the processing of impulses between components.

Plates may have different constitutions on their substrate. They may have only one layer of copper, two layers of copper, or multiple layers on both sides of the substrate.

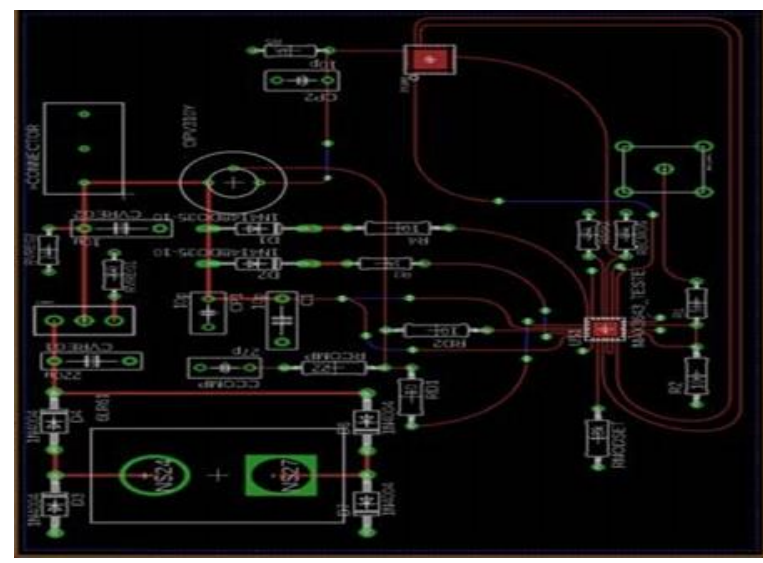

Fig. 9. Emitter PCB Project. 
As shown in Fig. 9 the final design for the printed circuit board of the circuit with the integrated components are responsible for the excitation and control of the laser, as well as the power circuit, responsible for feeding the integrated components.

\section{B. Optical Receiver}

For the realization of the optical receiver, the block diagram proposed is that represented in Fig. 10, which consists of the photodetetor SFH2701, followed by the first stage of amplification that is constituted by a transimpedance amplifier materialized by the integrated circuit MAX3654, being aided with a circuit of automatic gain control, by the amplifier OPA380, and finally the second stage of amplification with the amplifier THS4021 [18].

This circuit only constitutes the front-end of an optical receiver, and the issues of the demodulation of the received signals, as well as their decoding, have not been addressed in the base circuit of the basic optical receiver of this dissertation.

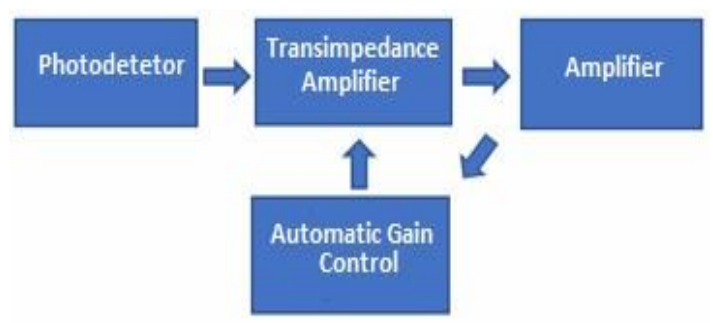

Fig. 10. Receiver diagram blocks.

\section{a) Photodiode $\mathrm{SFH} 2701$}

The receiver converts the received optical signal into an electrical signal. Photoelectric cells have the ability to transform light energy into electrical energy. The photoelectric effect occurs when a photon with sufficient energy reaches the diode, creating the electron-hole pair. If the absorption occurs in the region of depletion of the junction, the electrons are expelled from the junction by the electric field created in the depletion region. Thus, the holes move towards the anode, while the electrons in the direction of the cathode thus producing a photocurrent in the photodetector. The total current of the photodiode is the sum of the dark current (current that is generated in the absence of light) with the photocurrent originated from the photoelectric effect, so the dark current must be minimized to maximize the sensitivity of the photodiode [19].

The photodiode used was a photodiode of type SFH2701 from OSRAM Opto Electronics. This component receives the beam of light emitted by the laser emitter, converting it into an electric impulse. It has a maximum responsiveness of $0.5 \mathrm{~A} / \mathrm{W}$, with an incident wavelength of $780 \mathrm{~nm}$ and a visible red signal $(650 \mathrm{~nm})$, a responsiveness of $0.45 \mathrm{~A} / \mathrm{W}$. It has a maximum sensitivity for a wavelength of $820 \mathrm{~nm}$, which compared to our working length means that the photodetector is almost in the ideal situation with maximum responsiveness. Features a capacity of $3 \mathrm{pF}$. It shows good linearity when working in the photoconductive mode, introducing a very reduced black current in the signal, with the maximum value being introduced of $5 \mathrm{nA}$. This device operates efficiently from $-40{ }^{\circ} \mathrm{C}$ to $85^{\circ} \mathrm{C}$.

\section{b) Connections to the integrated MAX3654 and automatic gain control circuit}

After receiving the signal by the photodetector, it is preamplified. For that, a transimpedance amplifier was used which means that the conversion of the input current into a voltage at the output.

The Integrated MAX3654 is a highly linear and low noise transinpedance amplifier. Its gain (transimpedance) can be corrected between $43.5 \mathrm{~dB}$ and $62 \mathrm{~dB}$ at a voltage with an external control (VAGC pin). It can operate at working frequencies from $47 \mathrm{MHz}$ to $870 \mathrm{MHz}$, its maximum gain deviation is $\pm 0.75 \mathrm{~dB}$.

In the inputs (IN+ and IN-) and the outputs (OUT+ and OUT-) of the amplifier, each one of these pins are attributed one capacitor $\left(C_{1}, C_{2}, C_{3}\right.$ and $\left.C_{4}\right)$ of $1 \mathrm{nF}$ to filter possible continues components introduced by the circuit elements.

As for powering the circuit, and according to the specifications of the amplifier, need to place the MUTE, $V_{\mathrm{CC}} @ 1, V_{\mathrm{CC}} @ 4, V_{\mathrm{CC}} @ 9, V_{\mathrm{CC}} @ 12$ pins connected to a source having $5 \mathrm{~V}$ voltage and a current of $\left(I_{O P}\right)$ between 136 and $170 \mathrm{~mA}$.

Two levels of rust are present, namely the two components BLM15HD182 without any type of starting of the power to the photodiode in order to remove any high frequency noise originating in the voltage source.

The $V_{\mathrm{AGC}}$ pin defines the sweat gain through three switchable gain stages, each with a variable variable gain control. This produces a continuously variable variable gain, ranging from $62 \mathrm{~dB}$ (when $V_{A G C}=0.175 \mathrm{~V}$ ) to $43.5 \mathrm{~dB} \Omega$ (when $V_{A G C}=1.4 \mathrm{~V}$ ), given by Expression 10 .

$$
Z_{T}=62+20 \log \left[175 / v_{A G C}\right\rfloor 0.175 \leq v_{A C G} \leq 1.4
$$

$Z_{T}$ is the value of the transimpedance gain given in $\mathrm{dB} \Omega$, $V_{A G C}$ is the input value of the $V_{\mathrm{AGC}}$ pin in $\mathrm{mV}$.

For values less than $V_{A G C} \leq 0.175 \mathrm{~V}$ the gain is constant and maximum.

A set of comparators examines the input resistance value of the $V_{\mathrm{AGC}}$ pin and selects a range of values in the frame. Since the variables can vary, the output values, corresponding to $350 \mathrm{mV}$ and $700 \mathrm{mV}$, can be seen without output, which can lead to the interruption of the signal acquisition, as shown in Fig. 11.

\section{GAIN vs VOLTAGE CONTROL}

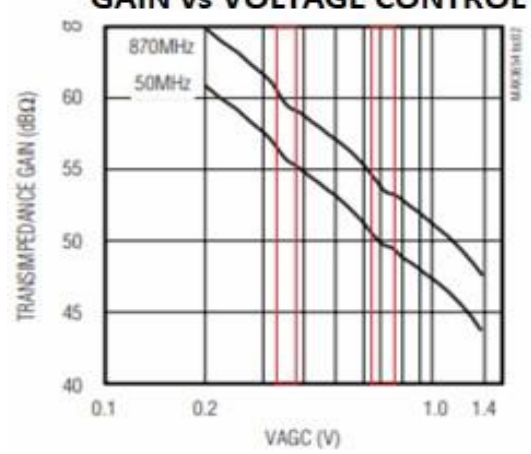

Fig. 11. Transimpedance gain according to voltage and frequency [28] 
The HYST pin allows controlling the hysteresis level in order to limit the drop in the transimpedance gain imposed by the transitions between the different stages of gain. The hysteresis level is controlled by the value of $R_{\text {HYST }}$ being that it is minimum $(0.13 \mathrm{~dB})$ when this pin is off, that is, in open circuit.

The MUTE pin must be supplied with a voltage greater than $0.8 \mathrm{~V}$ in order to operate in normal operation. When the power value of this pin is less than this value, the output signal is attenuated by $45 \mathrm{~dB}$.

As for the differential output of the amplifier, namely the OUT+ and OUT- pins, they must be connected to a balun transformer, namely the WBC1.1LB. The signal in OUT+ is in phase opposition to the signal in OUT-. Thus, with the use of a balun transformer, it is possible to invert the phase of the OUT- signal and make it possible to add both signals, which results in the output of the transformer, namely the pin SEC (pin 1) in a "clean" signal due to the phenomenon of destructive interference, which causes the cancellation of transmission noise.

As we have seen, we need a circuit that is responsible for gain control, that is, we need to implement a circuit that according to the value of the voltage received by the photodetector compensates the gain in order to keep the output of the amplification stage a constant value in order to avoid oscillations that could be detrimental to the detection of the information sent by the issuer.

For this, a circuit was designed with the amplifier, where the positive input is fed with the signal coming from the anode of the photodetector, through a resistance of $1 \mathrm{k} \Omega$ $\left(R_{6}\right)$. The negative input has the parallel of a capacitor $\left(C_{5}\right)$ and a resistor $\left(R_{5}\right)$, followed by a resistor $\left(R_{4}\right)$ in series, before connecting with the output of the amplifier.

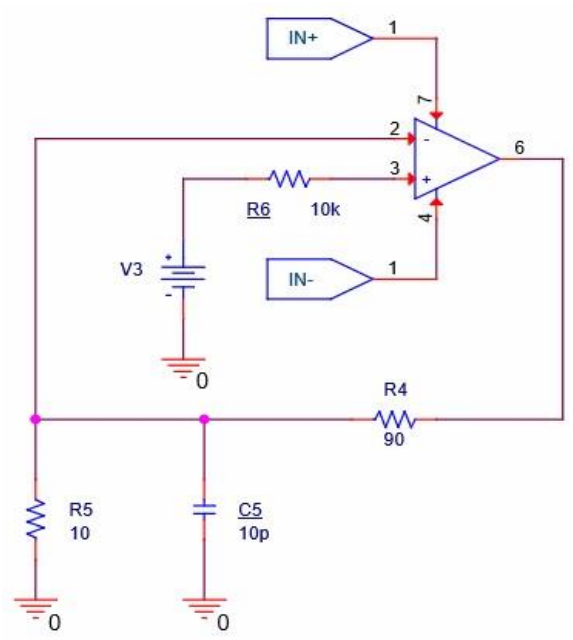

Fig. 12. Automatic gain control circuit.

\section{c) 2nd stage of amplification with amplifier THS4021}

In this way, we need to apply a second amplifier in order to increase the signal power to be demodulated and sampled later. To do this, we again use the THS4021ID amplifier with a combination of resistors, a can be seen in Fig. 13.

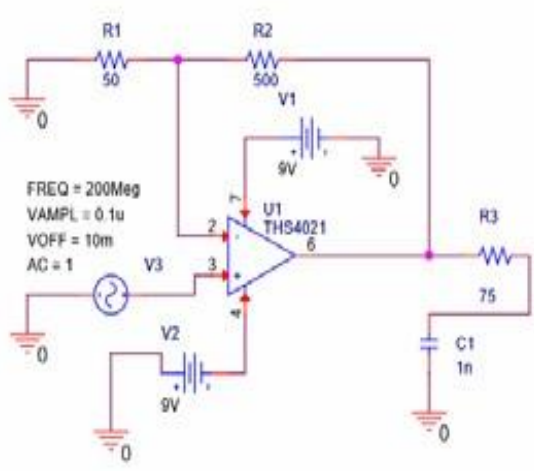

Fig. 13. Circuit with THS4021 amplifier.

In this circuit, $V_{3}$ represents the signal after passing through the WBC1.1LB transformer. The resistance $R_{3}$ represents the output of the optical receiver, namely the coaxial output of the system. The association of resistances $R_{1}$ and $R_{2}$ is responsible for calculating the gain of the amplification stage.

\section{d) Optical receiver circuit and PCB project}

Thus, the complete circuit of the optical receiver, with the photodetector, with the first stage of amplification and its respective automatic gain control circuit (amplifier OPA380) and with the second stage of amplification is represented by Fig. 14.

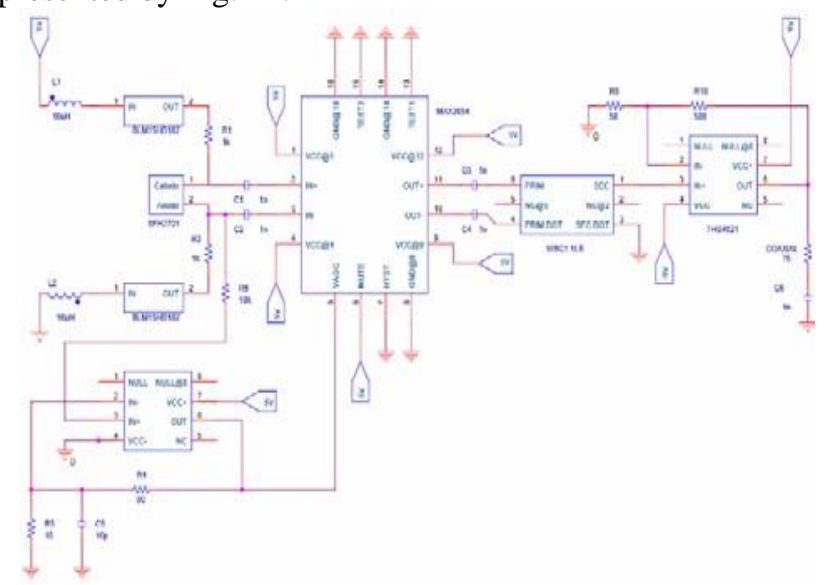

Fig. 14. Optical receiver circuit.

Is shown in Fig. 15 the PCB project for the printed circuit board of the circuit with the photodetector, the transimpedance amplifier with the automatic gain control and the second stage of amplification.

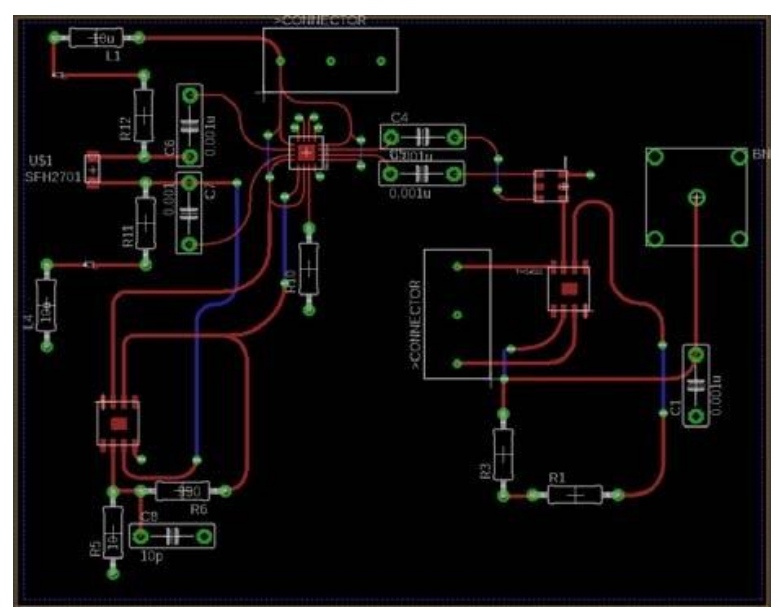

Fig. 15. Receiver PCB Project. 


\section{EXPERIMENTAL SiMULATIONS}

In this chapter we present some simulations of different blocks of the system. With the experimental simulations it is possible to obtain some results of the optical communication system and to validate the master's dissertation, comparing all the results with each other.

The programs used to perform the simulations were OrCAD Capture CIS - Lite and LTSpice XVII. There was a need to use two Spice simulators for two reasons, the first because OrCAD limits the number of nodes the circuit wiring can contain and the second because it had to resort to some specific built-in libraries provided by the manufacturers.

No simulations were performed at the level of the optical emitter blocks due to technical limitations. The libraries of the integrated MAX3643 and DS1865 are not available from the manufacturers, which did not allow the simulations with these components. A possible alternative would be to create the integrated libraries, but due to their high internal complexity of the electrical schematics of both components, not making an alternative feasible to achieve the simulations.

Regarding the optical receiver blocks, simulations were performed on the behavior of components THS4021 and OPA380 related to the automatic gain control circuit and also the behavior of component THS4021 relative to the second amplifier stage of the optical receiver. The simulation of the transimpedance amplifier had been considered, but for the same limitations presented previous, simulations of the behavior of the MAX3654 were not performed.

\section{A. Automatic Gain Control Circuit}

Before addressing the simulation question of the circuit that is responsible for automatic gain control in the transimpedance amplifier, it is important to recognize in what way, we can represent the influence that the predecessor blocks have on the block. In this case, it is important to point out the influence of the photodetector and the remaining set up on the gain control circuit.

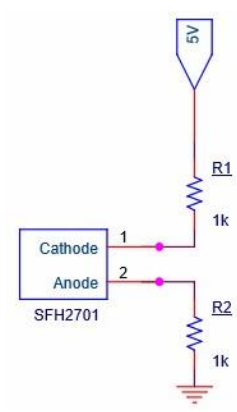

Fig. 16. Optical signal detection circuit.

When the photodetector does not receive any kind of excitation from outside, the circuit is simple to explain, with the formation of a current, which travels between the power supply $(5 \mathrm{~V})$ of the circuit and the ground. When the optical signals are detected by the photodetetor SFH2701, it causes a bias current of the photodiode, in the direction opposite to the current of the circuit, as we can see in Fig. 16, which will power the transimpedance amplifier to convert the voltage as already was reported. However, one way of representing these interactions in the gain control circuit is by placing a voltage source $\left(V_{3}\right)$ corresponding to the voltage drop in resistor $R_{2}$ and ground.

The simulations carried out were: DC Sweep with amplifiers OPA380 and THS4021, in order to verify which amplifier best fits the circuit of automatic gain control; the second floor of amplification simulations were based on the variation of the voltage at the output of the amplifier, with the application of different variables, namely, variation of the frequency of the input signal, temperature variation and finally, calculation of the gain at rest of the montage. Fig. 17 serves as an example of the results obtained in the simulations related to temperature variation.

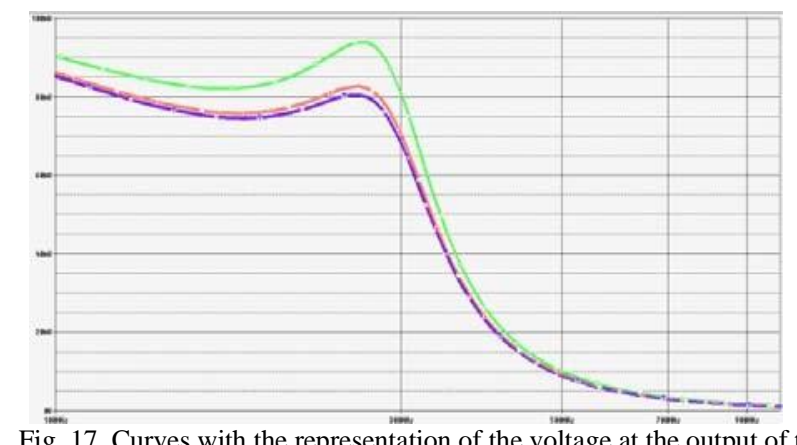

Fig. 17. Curves with the representation of the voltage at the output of the amplifier as a function of frequency - different temperatures.

\section{CONCLUSIONS}

To minimize the possible attenuations to suffer my optical communication system was defined that the wavelength of the system would be $\lambda=850 \mathrm{~nm}$, since this, minimizes the losses due to absorption in the particles suspended in the air.

As for the subsystem of the transmitter, an already existing circuit assembly was used with two integrated devices, the MAX3654 and the controller DS1865, which are responsible for controlling the excitation current of the laser and for adjusting that current, respectively, to maintain the luminous power at the output of the constant laser. To achieve the objective of the assembly it was necessary to correctly dimension the components connected to the pins of the integrated ones, since the laser must be correctly fed in current and controlled in temperature. A power circuit was implemented for all the components of this subsystem, as well as an alternative for tests in closed environments, using an external source to the subsystem. Having established all the elements and connections of the emitter circuit, the transmitter printed circuit board has been designed for subsystem validation.

The block diagram of an optical receiver was also presented, which served as the basis for the definition of the optical emitter of the dissertation. The receiver's focus was the front-end of a receiver, and there were no concerns regarding receiver reception of the signals. The result was a circuit consisting of the photodetetor PIN SFH2701, whose maximum sensitivity lies at the wavelength of the system, followed by a transformer amplifier MAX3654, with an automatic gain control circuit and finally a second stage of amplification consisting of the amplifier THS4021. To achieve the objective of the assembly it was necessary to correctly dimension all the components connected to the transimpedance amplifier, as well as the circuit responsible 
for the automatic gain control of the system and the second stage of amplification. Having established all the elements and connections of the receiver circuit, the receiver circuit board has been designed for subsystem validation.

In short, the realization of this work acquired competences in the design and realization of an optical communication system. This project proved to be multivalent, since in addition to the simulations carried out with a view to the design of the system, it also allowed to acquire practical skills in the realization of the same and allowed the use of several different tools for the design of printed circuits. In addition, it also allowed to consolidate knowledge that was obtained in the curricular units during the last years in the Academia Militar and in the Instituto Superior Técnico the modes.

This kind of circuits could be implemented in military vehicles, for example in UAVs [20]-[23] and could be powered by solar panels [24]-[32].

\section{REFERENCES}

[1] S. Tzu, The Art of War, USA: Harper Collins, 2011.

[2] K. Perusich, Information Warfare: Radar in World War II as an Historic Example, in International Symposium on Technology and Society, South Bend, USA, 1997.

[3] S. A. D. Kedan, Urban optical wireless communication networks: the main challenges and possible solutions, IEEE Communications (2004), S2-S7.

[4] G. Keiser, Optical Fiber Communications, McGraw-Hill International Editions - Second Edition.

[5] Hamamatsu, [Online]. http://www.hamamatsu.com/eu/en/community/

Available: automotive/interior/car_to_x.html. [Accessed 12 Outubro 2018].

[6] VICS, [Online]. Available: http://www.vics.or.jp/en/vics/ [Accessed 10 Outubro 2018].

[7] COOPERS, Co-operative Networks for Intelligent Road Safety Final report on demonstration, COOPERS integrated project, 2010.

[8] N. Kumar, Visible Light Communication Based Traffic Information Broadcasting Systems, International Journal of Future Computer and Communication 3 (2014), 26-30.

[9] A. K. A. S. Abrar Soudgar, Li-fi: An Infallible Standard for Future Indoor Communication, in International Conference on Electronics, Communication and Aerospace Technology (ICECA), 2017.

[10] I. I. A. E. K. Kim, Availability of Free Space Optics (FSO) and Hybrid FSO/RF Systems, Optical Access, Incorporated, 2002.

[11] J. Oscarsson, Simulation of Optical Communication for Formation Flying Spacecraft, Suécia, 2008.

[12] M. J. Barroso, O sistema de informação e comunicações tático (SIC-T) do Exército Português - Implicações doutrinárias, IESM, Lisboa, 2008.

[13] EDA, European Defence Agency, [Online]. Available: https://www.eda. europa.eu/docs/documents/Extract_from_NEC_Vision_Report.pdf . [Accessed 12 Outubro 2018].

[14] E. Português, Exército Português, [Online]. Available: https://www.exercito.pt/pt. [Accessed 12 Outubro 2018].

[15] MAX3643. [Online]. Available: https://datasheets.maximintegrated.com/en/ds/ MAX3643.pdf. [Accessed 12 Outubro 2018].

[16] J. Trindade, Sistema de comunicação ótica inter-satélites para aplicações em Defesa - I, Instituto Superior Técnico, Lisboa, 2016.

[17] T. Electronics, Laser OPV310, OPV310, OPV310Y, OPV314, OPV314Y, [Online]. Available: http://www.ttelectronics.com/sites/default/files/downloadfiles/OP V300-310Y-314Y_4.pdf. [Accessed 12 Outubro 2018].

[18] MAXIM, Maxim Integrated, MAXIM, [Online]. Available: https://datasheets. maximintegrated.com/en/ds/MAX3654.pdf [Accessed 12 Outubro 2018].

[19] M. \&. C. M. Vítor, Simulador de Receptor Óptico Digital de Modulação de Intensidade e Detecção Directa, Instituto Superior Técnico, Lisboa, 2009.
[20] Marques, Pedro, et al. "Communication Antenas for UAVs." Journal of Engineering Science and Technology Review 11.1 (2018): 90-102.

[21] Carneiro, António Fernando Alves, et al. "Smart Antenna forApplication in UAVs." Information, 9.12 (2018): 328.

[22] Gomes, Rui David Furtado Ribeiro, et al. "Study of a nano optical antenna for intersatellite communications." Optical and Quantum Electronics 49.4 (2017): 135.

[23] Torres, João Paulo N., et al. "The effect of shading on photovoltaic solar panels." Energy Systems 9.1 (2018): 195-208.

[24] Fernandes, Carlos AF, et al. "Cell string layout in solar photovoltaic collectors." Energy Conversion and Management 149 (2017): 997-1009.

[25] Fernandes, Carlos AF, et al. "Aging of solar PV plants and mitigation of their consequences." 2016 IEEE International Power Electronics and Motion Control Conference (PEMC). IEEE, 2016.

[26] Nashih, Samuel K., et al. "Validation of a simulation model for analysis of shading effects on photovoltaic panels." Journal of Solar Energy Engineering 138.4 (2016).

[27] Torres, João Paulo N., et al. "Effect of reflector geometry in the annual received radiation of low concentration photovoltaic systems." Energies 11.7 (2018): 1878.

[28] Alves, Pedro, et al. "From Sweden to Portugal: The effect of very distinct climate zones on energy efficiency of a concentrating photovoltaic/thermal system (CPV/T)." Solar Energy 188 (2019): 96-110.

[29] Campos, Catarina Sofia, João Paulo N. Torres, and João FP Fernandes. "Effects of the heat transfer fluid selection on the efficiency of a hybrid concentrated photovoltaic and thermal collector." Energies 12.9 (2019): 1814.

[30] Marques, Luís, João Paulo N. Torres, and PJ Costa Branco. "Triangular shape geometry in a Solarus $\mathrm{AB}$ concentrating photovoltaic-thermal collector." International Journal on Interactive Design and Manufacturing (IJIDeM) 12.4 (2018): $1455-1468$.

[31] Gomes, João, et al. "Analysis of different C-PVT reflector geometries." 2016 IEEE International Power Electronics and Motion Control Conference (PEMC). IEEE, 2016.

Fernandes, Carlos AF, et al. "Stationary solar concentrating photovoltaic-thermal collector-cell string layout." 2016 IEEE International Power Electronics and Motion Control Conference (PEMC). IEEE, 2016. 